\title{
InSb Quantum Dots in an InAsSb Matrix Grown by Molecular Beam Epitaxy
}

\author{
A.N. Semenov*, V.A. Solov'ev, B.Ya. Meltser, \\ O.G. Lyublinskaya, Ya.V. Terent'ev, A.A. Sitnikova \\ AND S.V. IVANOV \\ Ioffe Physico-Technical Institute \\ 26 Polytekhnicheskaya str., St. Petersburg 194021, Russia
}

\begin{abstract}
We report on molecular beam epitaxy of InSb insertions in InAs and InAsSb matrices, emitting at wavelengths beyond $4 \mu \mathrm{m}$. Different growth techniques for deposition of InSb quantum dots in the 1-2 monolayer range of the InSb nominal thickness, namely conventional molecular beam epitaxy and migration enhanced epitaxy, as well as different matrices (InAs and InAsSb) have been employed for increasing the emission wavelength of the $\mathrm{InSb} / \mathrm{InAs}$ nanostructures. The formation of InSb quantum dots has been studied in situ using reflection high energy electron diffraction and ex situ by using transmission electron microscopy. The peculiarities of $\operatorname{In}(\mathrm{Ga}) \mathrm{AsSb}$ alloys growth and compositional control are also discussed. Bright photoluminescence up to $4.5 \mu \mathrm{m}$ has been observed at $80 \mathrm{~K}$.
\end{abstract}

PACS numbers: 78.55.Cr, 78.67.Hc, 81.07.Ta, 81.15.Hi, 81.16.Dn

\section{Introduction}

InSb and $\mathrm{InAs}_{y} \mathrm{Sb}_{1-y}$ ternary alloys are highly promising materials for different mid-infrared (mid-IR) devices. The $\mathrm{InAs}_{y} \mathrm{Sb}_{1-y}$ alloys with a Sb content near 9\% (lattice matched to GaSb) are widely used for light emitting diodes [1] and low-cost room-temperature detectors [2]. The $\mathrm{InAs}_{y} \mathrm{Sb}_{1-y}$ alloys with the high Sb content are used for Hall-sensors [3] and as cooled far-IR detectors [4]. Unlike the InAsSb alloys, there are only a few reports of devices based on InSb quantum dots (QDs), although InSb QDs embedded in different matrices have been studied. In particular, self-assembled InSb QDs have been fabricated in GaAs [5, 6],

*corresponding author; e-mail: semenov@beam.ioffe.ru 
GaSb $[7,8]$, and InP [9] matrices. In these papers only low-temperature photoluminescence (PL) in the near-IR spectral range from QDs nanostructures was reported, and the longest PL wavelength of $1.7 \mu \mathrm{m}$ (at $77 \mathrm{~K}$ ) was observed from the $\mathrm{InSb} / \mathrm{GaSb}$ nanostructures.

Recently, we have reported on molecular beam epitaxial (MBE) growth of InSb sub-monolayers (SMLs) embedded in the InAs matrix [10], which demonstrate bright PL in the 3.4-4.3 $\mu \mathrm{m}$ wavelength range up to the room-temperature [11]. The SML insertions with the InSb nominal thickness ranged from 0.6 up to 1 monolayer (ML) as dependent on growth temperature $\left(T_{\mathrm{S}}=\right.$ $430-485^{\circ} \mathrm{C}$ ) were formed by a few second exposure of the InAs growth surface to the antimony flux with no intentional deposition of InSb. The laser emission at a wavelength of $3.08 \mu \mathrm{m}(T=60 \mathrm{~K})$ under pulse injection pumping has been demonstrated in a hybrid $p$-AlGaAsSb/InAs $/ n$-CdMgSe double heterostructure laser diode with the multiple type II InSb/InAs nanostructures in the active region [12].

In this paper we study different possibilities to increase PL wavelength in the InSb/InAs nanostructures. We employed (i) the narrower gap matrix InAsSb as well as (ii) increasing the nominal thickness of the InSb insertion above $1 \mathrm{ML}$ in InAs, i.e. the mean size of InSb QDs, by intentional deposition of InSb, using both conventional MBE and migration enhanced epitaxy (MEE) techniques. The MBE growth, structural and PL properties of the InSb/InAs and InSb/InAsSb QD nanostructures are reported.

\section{Growth procedure}

The samples were grown on GaSb and InAs (001) substrates using a RIBER $32 \mathrm{P}$ setup. Conventional solid source effusion cells were used to produce $\mathrm{In}, \mathrm{Al}$, and $\mathrm{Sb}_{4}$ fluxes, whereas $\mathrm{As}_{4}$ flux was supplied from a VAC-500 valved cracking cell. A substrate temperature $T_{\mathrm{S}}$ was measured by an IR pyrometer calibrated by different known surface reconstruction transitions on GaAs and GaSb surface, monitored in situ by reflection high energy electron diffraction (RHEED).

A large set of InAsSb and GaInAsSb alloy layers with different Sb content was grown at $T_{\mathrm{S}} \sim 430^{\circ} \mathrm{C}$ for investigation of $\mathrm{Sb}$ incorporation behavior.

An active region of the structures with InSb QDs consists of a $0.4 \mu \mathrm{m}$ thick InAs or InAsSb layer centered with an InSb insertion. The initial stage of InSb QD formation includes the procedure of the InAs growth surface exposure to the $\mathrm{Sb}_{4}$ flux, described in [10], followed by a short-time growth interruption (GI). The second stage comprises the deposition of an additional amount of InSb by either conventional MBE or MEE. In both cases, the In flux intensity was kept the same, corresponding to the InSb growth rate of $\sim 0.1 \mathrm{ML} / \mathrm{s}$. The growth temperature was $430^{\circ} \mathrm{C}$ as well.

The RHEED specular spot intensity (SSI) oscillation technique was used to study in situ the InSb QD formation. Transmission electron microscopy (TEM) 
studies were performed using an EM-420 Philips microscope operating at $100 \mathrm{keV}$. PL spectra were measured under a diode laser excitation $\left(\lambda_{\text {ex }}=809 \mathrm{~nm}\right)$ at the $\sim 1.5 \mathrm{~W} / \mathrm{cm}^{2}$ excitation power density and temperature tuning in the $15 \div 300 \mathrm{~K}$ range. Emitted light was detected with a nitrogen-cooled InSb photodiode and a standard lock-in amplification technique.

\section{Result and discussion}

The incorporation rate of $\mathrm{Sb}$ molecules was studied during the growth of InAsSb and GaInAsSb alloys for small Sb contents (up to 15\%) and high Sb contents (about 90\%), respectively. The growth rate (incorporation rate) of group III elements was identical for both alloys as well as the incident As flux and $T_{\mathrm{S}}$, that provides similar conditions for $\mathrm{Sb}$ incorporation in these alloys.

The calculated and experimental dependences of $\mathrm{Sb}$ content $(y)$ on the ratio of $\mathrm{Sb}$ atomic flux evaluated from the $\mathrm{Sb}_{4}$ beam equivalent pressure (BEP) to the total group V flux (As BEP + Sb BEP) are shown in Fig. 1. Calculations in the framework of a thermodynamic model (solid lines in Fig. 1) predict a substantial dominance of arsenic in the solid alloys even at low incident As flux. In contrast, the experimental dependence (solid circles and dashed line) shows that the incorporation of arsenic into the alloys is significantly suppressed in comparison with antimony. These results are in good agreement with the data on Sb incorporation in the AlGaAsSb alloys [13]. For low Sb content the $\mathrm{In}(\mathrm{Ga}) \mathrm{AsSb}$ alloy composition linearly depends on the Sb BEP, similar to that reported recently by Marcadet et al. [1]. At higher Sb fluxes, the dependence saturates, confirming previously published data [14]. These results show that the $\mathrm{In}(\mathrm{Ga}) \mathrm{AsSb}$ alloy composition can be most efficiently controlled by changing the Sb flux at fixed group III and

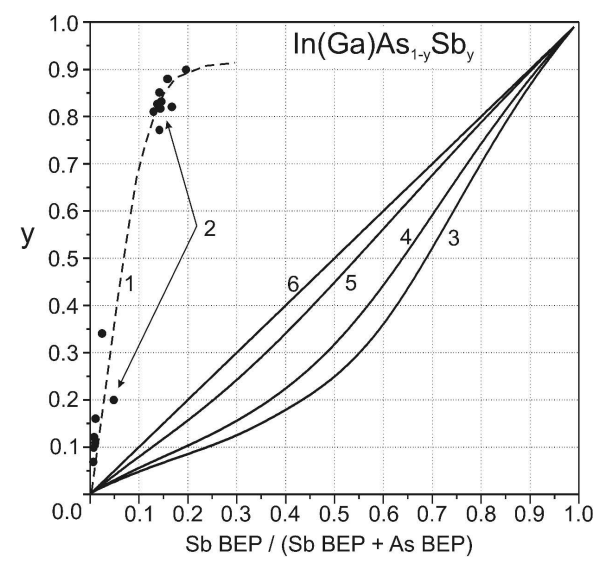

Fig. 1. Sb content in $\operatorname{In}(\mathrm{Ga})$ AsSb alloys as a function of group $\mathrm{V}$ flux ratio (BEP $\mathrm{Sb} / \mathrm{BEP} \mathrm{As}+\mathrm{BEP} \mathrm{Sb}$ ): (1) dashed curve connecting the experimental points at the growth temperature $430^{\circ} \mathrm{C}$; (2) experimental data; (3-6) thermodynamic predictions at $T_{\mathrm{S}}$ equal $530,520,510^{\circ} \mathrm{C}$ and below $450^{\circ} \mathrm{C}$, respectively. 


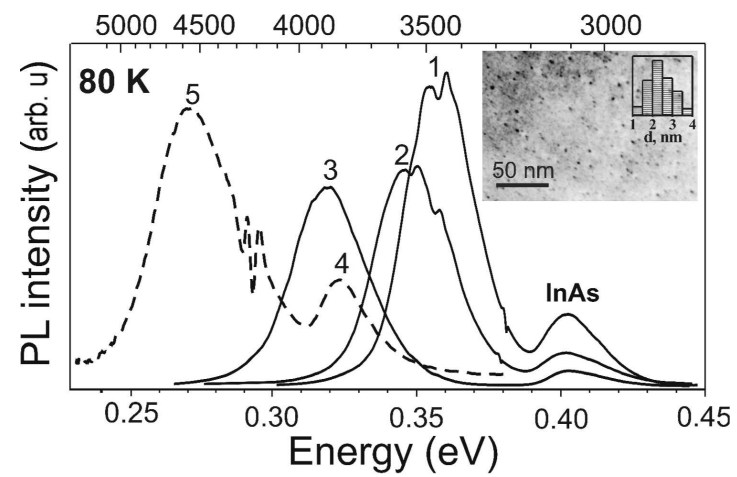

Fig. 2. PL spectra $(80 \mathrm{~K})$ of $\mathrm{InSb} / \mathrm{InAs}$ nanostructures grown by different techniques in InAs matrix (solid curves) and InAsSb matrix (dashed curve). The peak attribution is discussed in the text.

As fluxes and $T_{\mathrm{S}}$. The $\mathrm{InAs}_{0.91} \mathrm{Sb}_{0.09}$ alloys lattice-matched to GaSb demonstrate bright PL (peak \#4 at the dashed line in Fig. 2) and high structural quality. Their exploitation as a matrix in the InSb/InAs nanostructures allows fabrication of InSb QDs on GaSb substrates.

The PL spectra of $\sim 1 \mathrm{ML}$ InSb insertions in the $\mathrm{InAs}_{0.91} \mathrm{Sb}_{0.09}$ matrix (dashed line, peak \#5) as well as the InAs (solid line, peak \#1) matrix, deposited under the same conditions ( $\mathrm{Sb}$ flux, shutter sequence and $T_{\mathrm{S}}=430^{\circ} \mathrm{C}$ ) are shown in Fig. 2. The narrower gap matrix (InAsSb) results in a significant $(\sim 1 \mu \mathrm{m})$ red shift of the InSb QD related peak. Although no intentional InSb deposition was made in both cases, the dense array of InSb QDs was revealed in plan view TEM images, as shown in the inset to Fig. 2 for the InSb/InAs structure. A lateral size of the QDs and their sheet density are estimated as approximately $2.5 \mathrm{~nm}$ and $10^{12} \mathrm{~cm}^{-2}$, respectively.

To study the kinetics of InSb QDs formation during deposition of an additional (extra-ML) amount of InSb in the InAs matrix by MEE and MBE, the RHEED oscillation technique was used. The RHEED SSI oscillations for different growth technique of InSb deposition are presented in Fig. 3.

The introduction of GI after the exposure of the InAs surface to the Sb flux results in the dramatic decrease in the SSI intensity (Fig. 3a). In our opinion, such unusual behavior of SSI under the anion stabilization conditions may be explained by an onset of the self-organization of InSb-enriched islands during the GI. It is expected that a certain duration of the GI exists that corresponds to the most uniform QD array. Nevertheless, RHEED pattern exhibits the $(3 \times 1)$ Sb-stabilized reconstruction without $3 \mathrm{D}$ features that confirms a very small size of the InSb QDs.

The SSI oscillations during the deposition of an additional amount of InSb using MEE are presented in Fig. 3b. Deposition of In is assumed to lead to completing the InSb ML. One can distinguish the corresponding half-period In-induced oscillation. Another half-period As-induced oscillation occurs after the 


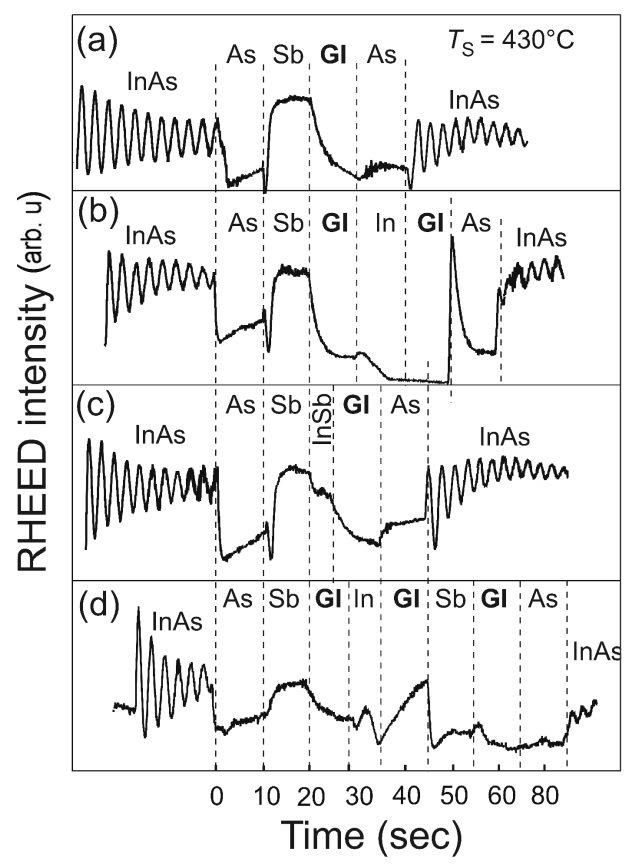

Fig. 3. RHEED oscillations during formation of single InSb insertion by: (a) exposure of an InAs surface to the $\mathrm{Sb}_{4}$ flux only; (b) the exposure followed by In deposition, (c) the exposure followed by InSb MBE deposition and (d) the exposure followed by completed MEE cycle.

As shutter is opened. Such a procedure of the additional In deposition results only in a small shift of the InSb-QD-related PL peak to longer wavelengths, as compared to the PL band maximum of $\sim 1 \mathrm{ML}$ InSb grown as monitored in Fig. 3a (Fig. 2, peaks $\# 2$ and $\# 1$, respectively). To increase the nominal thickness of deposited InSb beyond $1 \mathrm{ML}$ and, thus, the mean size of the InSb QDs, either a complete MEE cycle (consequent deposition of In and Sb atoms) or MBE should be used.

The RHEED SSI oscillations for the case of complete MEE growth of InSb are presented in Fig. 3d. In addition to the RHEED SSI decrease during GI after the Sb exposure, observed earlier in Fig. 3a, the next dramatic decrease in SSI occurs immediately after the beginning of the second Sb exposure. The observed decrease in RHEED SSI appears to be due to the growth surface which becomes more rough. This indicates that at this stage QDs have already formed, and the Sb exposure leads to increasing the size of QDs. No half-period As-induced oscillation appears here when the As shutter is opened in contrast to the RHEED SSI oscillations in Fig. 3b. Its means that all deposited In atoms have been encapsulated into the InSb islands and the total amount of InSb is about 1.5 ML. This QD morphology variation is accompanied by the strong red shift of the InSb PL line maximum, as shown in Fig. 2 (peak \#3). 
The RHEED SSI oscillations for the case of conventional MBE growth of InSb followed after the InAs exposition to the Sb flux are presented in Fig. 3c. There is an important difference between the MBE and MEE (Fig. 3b) cases, namely, the 3D-related features appear in the RHEED pattern during the InSb MBE deposition. One should especially note that MBE deposition of InSb without preliminary exposure of an InAs surface to the Sb flux is non-reproducible and the resultant InSb-related emission wavelength is poorly controlled.

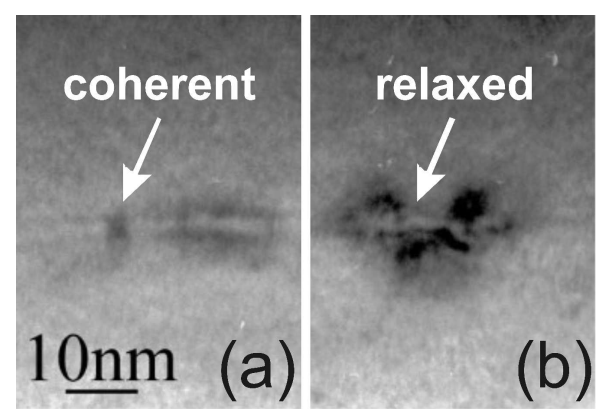

Fig. 4. Cross-sectional TEM images of $2 \mathrm{ML}$ InSb insertion into InAs grown by conventional MBE technique: (a) coherent, (b) relaxed.

TEM images of the InSb insertions grown by MBE technique show that intentional deposition of InSb results in enlarging the QDs lateral sizes. If the InSb nominal thickness exceeds $2 \mathrm{ML}$, the growth of non-coherent inclusions is observed. Figure 4 presents the cross-section images of the InSb/InAs sample with the $2 \mathrm{ML}$ insertion thickness. In the images two types of diffraction contrast are revealed. Spherical dark spot in the cross-sectional image (Fig. 4a) originates from the coherent InSb islands. Another disk-shaped island shown in the TEM image (Fig. 4b) is significantly larger in a diameter and surrounded by defects generated at the island/matrix interface. The elaboration of defects indicates the beginning of the strain relaxation process and formation of non-coherent islands within the $2 \mathrm{ML}$ insertion. A characteristic lateral size of the islands and their sheet density derived from the analysis of plain-view images of the sample, are evaluated as approximately $25 \mathrm{~nm}$ and $10^{10} \mathrm{~cm}^{-2}$, respectively. The array density of coherent islands with lateral size $\sim 15 \mathrm{~nm}$ is extremely low and estimated as about $10^{9} \mathrm{~cm}^{-2}$.

\section{Conclusion}

In summary, we have grown by MBE InSb QDs in InAs matrix with extra-monolayer InSb insertions formed by different growth techniques including (i) an exposure of the InAs growth surface to the $\mathrm{Sb}_{4}$ flux and (ii) MEE or MBE deposition of an additional amount of InSb. Both MBE and complete MEE techniques result in a pronounced red shift of the InSb-related PL band. Even the more 
strong red shift (beyond $4.5 \mu \mathrm{m}$ at $80 \mathrm{~K}$ ) has been achieved for a $\sim 1 \mathrm{ML}$ InSb insertion in the $\mathrm{InAs}_{0.91} \mathrm{Sb}_{0.09}$ matrix, formed by only the $\mathrm{Sb}_{4}$ exposure at $430^{\circ} \mathrm{C}$. An importance of growth interruptions after the exposure procedure has been discussed using a detailed analysis of RHEED SSI oscillations. The $\operatorname{InSb} / \operatorname{InAs}(\mathrm{Sb})$ sub-monolayer and extra-monolayer QD nanostructures are supposed to be promising candidates for the active region of mid-IR laser diodes emitting in the whole $3-5 \mu \mathrm{m}$ range.

\section{Acknowledgment}

The work was partly supported by RFBR and the Program of the Physical Science Department of RAS. S.V.I. acknowledges the support of RSSF.

\section{References}

[1] X. Marcadet, A. Racovska, I. Prevot, G. Glastre, B. Vinter, V. Berger, J. Cryst. Growth 227-228, 609 (2001).

[2] A. Wilk, B. Fraisse, P. Christol, G. Boissier, P. Grech, M.El. Gazouli, Y. Rouillard, A.N. Baranov, A. Joullie, J. Cryst. Growth 227-228, 586 (2001).

[3] G. Kida, III-Vs Rev. 16, 24 (2003).

[4] H. Simchi, III-Vs Rev. 17, 27 (2004).

[5] B.R. Bennett, R. Magno, B.V. Shanabrook, Appl. Phys. Lett. 68, 505 (1996).

[6] E.R. Glaser, B.R. Bennett, B.V. Shanabrook, R. Magno, Appl. Phys. Lett. 68, 3614 (1996).

[7] A.F. Tsatsul'nikov, N.N. Ledentsov, M.V. Maximov, B.Ya. Meltser, P.V. Neklyudov, S.V. Ivanov, B.V. Volovik, A.K. Kryganovskii, A.A. Suvorova, A.N. Titikov, P.S. Kop'ev, Zh.I. Alferov, M. Grundmann, D. Bimberg, J. Electron. Mater. 27, 414 (1998).

[8] A.F. Tsatsul'nikov, D.A. Bedarev, B.V. Volovik, S.V. Ivanov, M.V. Maximov, Yu.G. Musihin, N.N. Ledentsov, B.Ya. Meltser, V.A. Solov'ev, P.S. Kop'ev, A.Yu. Chernyshov, M.V. Belousov, Semiconductors 33, 886 (1999).

[9] T. Utzmeier, G. Armelles, P.A. Postigo, F. Briones, Phys. Rev. B 56, 3621 (1997).

[10] S.V. Ivanov, A.N. Semenov, V.A. Solov'ev, O.G. Lyublinskaya, B.Ya. Meltser, D.D. Solnyshkov, L.G. Prokopova, A.A. Sitnikova, A.A. Toropov, P.S. Kop'ev. J. Cryst. Growth 278, 72 (2005).

[11] V.A. Solov'ev, O.G. Lyublinskaya, B.Ya. Meltser, A.N. Semenov, D.D. Solnyshkov, A.A. Toropov, S.V. Ivanov, P.S. Kop'ev, Appl. Phys. Lett. 86, 011109 (2005).

[12] V.A. Solov'ev, I.V. Sedova, O.G. Lyublinskaya, A.N. Semenov, B.Ya. Meltser, S.V. Sorokin, Ya.V. Terent'ev, S.V. Ivanov, Tech. Phys. Lett. 31, 235 (2005).

[13] A.N. Semenov, V.S. Sorokin, V.A. Solov'ev, B.Ya. Mel'tser, S.V. Ivanov, Semiconductors 38, 266 (2004).

[14] G.S. Lee, Y. Lo, Y.F. Lin, S.M. Bedair, W.D. Laidig, Appl. Phys. Lett. 47, 1219 (1985). 